\title{
A Synthesis of High Purity Single-Walled Carbon Nanotubes from Small Diameters of Cobalt Nanoparticles by Using Oxygen-Assisted Chemical Vapor Deposition Process ${ }^{\dagger}$
}

\author{
Hye Ryung Byon, Hyunseob Lim, Hyun Jae Song, and Hee Cheul Choi ${ }^{*}$ \\ Department of Chemistry, Pohang University of Science and Technologv, Pohang 790-784, Korea. "E-mail: choihc@postech.edu \\ Received June 7, 2007
}

\begin{abstract}
$\Lambda$ successful combination of "oxygen-assisted chemical vapor deposition (CVD) process" and Co catalyst nanoparticles to grow highly pure single walled carbon nanotubes (SWNTs) was demonstrated. Recently, it was reported that addition of small amounts of oxygen during CVD process dramatically increased the purity and yield of carbon nanotubes. I Iowever, this strategy could not be applied for discrete Fe nanoparticle catalysts from which appropriate yields of SWNTs could be grown directly on solid substrates, and fabricated into field effect transistors (FETs) quite efficiently. The main reason for this failure is due to the carbothermal reduction which results in $\mathrm{SiO}_{2}$ nanotrench formation. We found that the oxygen-assisted CVD process could be successfully applied for the growth of highly pure SWNTs by switching the catalyst from Fe to Co nanoparticles. The topological morphologies and p-type transistor electrical transport properties of the grown SWNTs were examined by using atomic force microscope ( $\Lambda F M)$, Raman, and from FET devices fabricated by photolithography.
\end{abstract}

Key Words : Cobalt nanoparticle, Oxygen-assisted chemical vapor deposition, Single-walled carbon nanotubes.

\section{Introduction}

Carbon nanotube is one of the exclusively studied one dimensional nanoscale materials as it has unique properties that can be applied for chemical and biochemical sensors, ${ }^{1(a) ! b)}$ energy storage, ${ }^{1(c)}$ field emission display ${ }^{1(d)}$ and field effect transistor, ${ }^{\text {lec }}$ etc. As one of the requirements for the successful attainment of such applications, clean and less defective carbon nanotubes are demanded to be synthesized. During the growth of carbon nanotubes, amorphous carbons and unnecessary metal-carton composites are naturally produced, which are accountable for the high impurity contents and low yield of carbon nanotubes due to the catalyst poisoning effect. ${ }^{2} E x$ sith chemical purification treatments effectively eliminate metal dusts, ${ }^{3}$ but such methods also accompany chemical damages on the surfaces of carbon nanotubes.

Recently, oxygen-involved growth methods were reported as they effectively remove amorphous carbons during the nanotube growth. Moreover, oxygen-environment induces re-activation of poisoned catalyst nanoparticles, resulting in very high yield of single walled carbon nanotubes (SWNTs) with high purity. ${ }^{4}$ In both cases, Fe thin film was used as a core catalyst. However, it was difficult to adapt this strategy for the direct synthesis of 'moderately high yield" of SWNTs on a $\mathrm{Si}_{2} \mathrm{SiO}_{2}$ substrate which is prototypical to the fabrication of field effect transistor (FET) devices. Actually, when the similar strategy was applied to highly dense discrete Fe nanoparticles for the growth of SWNTs by oxygen-assisted chemical vapor deposition (CVD) process,

${ }^{\dagger}$ This paper is dedicaled to Professor Sang Chul Shim in honor of his retirement. thermochemical etching of $\mathrm{SiO}_{2}$ layer occurred instead of highly pure SWNT formation due to the thermocatalytic reduction of $\mathrm{SiO}_{2}$ by $\mathrm{Fe}$ nanoparticles. ${ }^{6}$

In this work, we demonstrate the utilization of Co catalyst nanoparticles for the growth of pure SWNTs in oxygenassisted CVD condition. The effect of oxygen determining the purity of resulting SWNTs was confirmed by comparative experiments performed in conventional thermal CVD growth condition. The narrow size distributions of $\mathrm{Co}$ catalyst nanoparticles as well as of the resulting SWNTs were confirmed by atomic force microscope (AFM). Raman spectra and electrical analysis revealed that the synthesized SWNTs were less defective, and that they showed typical ptype semiconducting transport properties, respectively.

\section{Experimental}

For the formation of cobalt nanoparticles on a $\mathrm{Si} / \mathrm{SiO}_{2}$ substrate, a catalyst solution was prepared by dissolving 0.01 wt $\%$ of cobalt(II $)$ acetate tetralhydrate $\left(\left(\mathrm{CH}_{3} \mathrm{COO}\right)_{2} \mathrm{Co} \cdot 4 \mathrm{H}_{2} \mathrm{O}\right.$, $99.999 \%$, Aldrich) in ethanol under ultrasonication (Fisher Scientific, FS60, 100W, $42 \mathrm{kHz} \pm 6 \%$ ). A precleaned Si $\mathrm{SiO}_{2}$ substrate (thermally grown $500 \mathrm{~nm}$ thick amorphous $\mathrm{SiO}_{2}$ layer on highly doped p-type $\mathrm{Si}<100>$ ) was immersed into the catalyst solution for $10 \mathrm{~min}$. The $\mathrm{Si}_{2} \mathrm{SiO}_{2}$ substrate was then dried by $\mathrm{N}_{2}$ gas and calcined in air at $400^{\circ} \mathrm{C}$ for 5 $\mathrm{min}^{7}{ }^{7}$ The population of Co nanoparticles on the substrate was controlled by sonication time. Normally, less than 2 hours of sonication results in low population of $\mathrm{Co}$ nanoparticles while high population of Co nanoparticles was derived from longer than 3 hours of sonication.

The calcined substrate was then transferred to a quartz tube in CVD system and heated up to $900{ }^{\circ} \mathrm{C}$ under a 500 
sccm of $\mathrm{H}_{2}$ gas flow environment. When temperature was reached to $830^{\circ} \mathrm{C}, 1000 \mathrm{sccm}$ of $\mathrm{CH}_{4}$ and $20 \mathrm{sccm}$ of $\mathrm{C}_{2} \mathrm{H}_{4}$ gases were introduced. In oxygen-assisted CVD process case, $1.5 \mathrm{sccm}$ of $\mathrm{O}_{2}$ gas was introduced at $830^{\circ} \mathrm{C}$. After 10 min of reaction at $900^{\circ} \mathrm{C}$, all gases were turned off except $\mathrm{H}_{2}$ to cool down the system.

Atomic force microscope (AFM, NanoScope IIIa, Digital Instruments) was used to characterize the topographical morphologies of Co nanoparticles and SWNTs on the Si/ $\mathrm{SiO}$ substrate. Raman spectroscopy was measured with Renishaw RM 1000-Invia at the condition of $20 \mathrm{~mW}$ of excitation power and $632.8 \mathrm{~nm}(1.96 \mathrm{eV})$ of laser wavelength. SWNT-FETs were fabricated using a photolithography ${ }^{\gamma}$ and their electronic properties were measured at room temperature by using a semiconductor analyzer (Keithley, 4200-SCS).

\section{Results and Discussion}

In contrast to the most popularly used Fe precursors, cobalt(II) acetate tetrahydrate showed difficulty in forming a homogeneous solution in water or ethanol at room temperature because of its low solubility. The solubility of Co precursor in ethanol was substantially improved when ultrasonic power was applied. We observed well dispersed individual Co nanoparticles on a $\mathrm{Si} / \mathrm{SiO}_{2}$ substrate when it was immersed into a catalyst solution which was treated for 2 hours of sonication followed by calcination in air at $400^{\circ} \mathrm{C}$ (Figure I(a)). A very narrow size distribution of Co nanoparticles having 0.6 to $1.6 \mathrm{~mm}$ diameters was obtained from AFM height profile measurements (Figure 1(b)). The average diameter of $0.88 \pm 0.20 \mathrm{~nm}$ is much smaller than the average diameter of $\mathrm{Fe}$ catalyst nanoparticles previously prepared by using ferritin, ${ }^{2}$ dendrimer, ${ }^{9,(j)}$ hydroxylamine, ${ }^{9(b)}$ or phosphoric acid. ${ }^{9 c i}$ We found that the density of $\mathrm{Co}$ nanoparticle was closely related to the sonication time. Regardless of the immersing time of $\mathrm{Si} / \mathrm{SiO}_{2}$ substrate into the catalyst solution, much higher density of Co nanoparticle was obtained from the solution sonicated for $3 \mathrm{~h}$ than from the one sonicated for $2 \mathrm{~h}$. This result indicates that $\mathrm{Co}$ nanoparticles are mostly prepared during the sonication
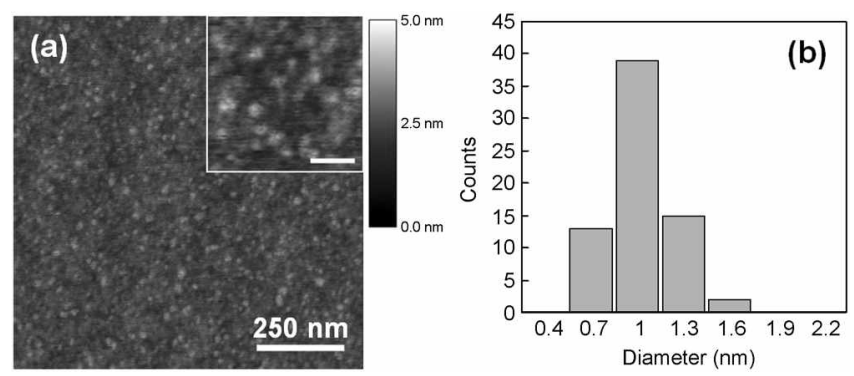

Figure 1. (a) AFM image of Co nanoparticles on $\mathrm{Si}_{2} \mathrm{SiO}_{2}$ substrate. The scale of $z$ axis is 0 to $5 \mathrm{~nm}$. Inset is a high magnification image of low yield of Co nanoparticles. $\Lambda$ scale bar indicates 50 nun and $z$ axis is 0 to 3 nun. (b) $\Lambda$ histogram of the diameter distribution of $\mathrm{CO}$ nanoparticles on the substrate. The measured average diameter is $0.88 \pm 0.20 \mathrm{~nm}$.
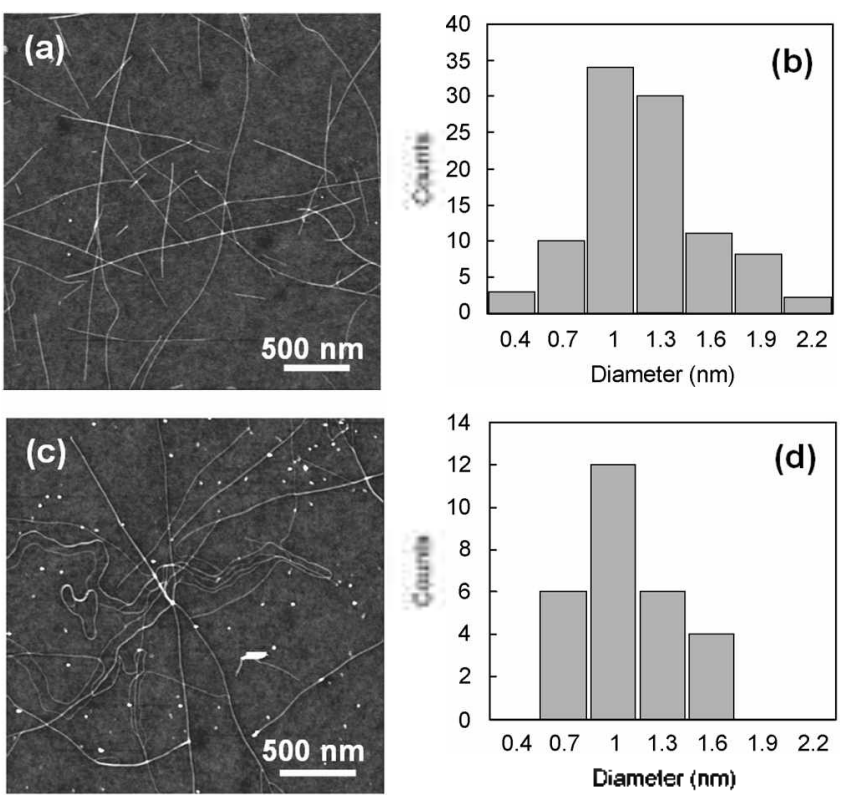

Figure 2. $A F M$ images of SWNTs grown from Co nanoparticles made by (a) oxygen-assisted CVD and (c) thermal CVD process. (b, d) Diameter distributions of SWNTs of (a) and (c) are shown, respectively.

process, and the yield is higher upon longer treatment.

Using low density Co catalyst coated substrates, SWNTs were attempted to grow under both oxygen-assisted CVD and conventional thennal CVD conditions. Figure 2 shows that SWNTs are grown successfully both by oxygen-assisted CVD process (Figure 2(a)) and themal CVD process (Figure 2(c)). The diameter distributions of both SWNTs are similar each other (Figure 2(b) and 2(d)), of which average diameters are $1.06 \pm 0.38$ and $0.93 \pm 0.29 \mathrm{~nm}$, respectively. We concluded that SWNTs were synthesized from individual Co nanoparticles based on the facts that the diameters of SWNTs are highly corresponding to those of catalyst nanoparticles (Figure I(b) vs. Figure 2(b) or (d)), and that most of SWNTs have less than $2 \mathrm{~nm}$ of diameters. The degree of purity, a strategic concem of current research, can be macroscopically decided by 1 ) the amounts of amorphous carbons randomly coated (or deposited) on the sidewalls of carbon nanotubes, and 2) the presence and population of unreacted catalyst nanoparticles on the substrates. It was difficult to ascertain any distinct difference in the amounts of amorphous carbons on SWNTs grown by either oxygenassisted CVD or thermal CVD. However, the population of unreacted Co catalyst nanoparticles left on the substrate after the growth was significantly distinguishable: in contrast to the thermal CVD process, most of unreacted catalyst nanoparticles were removed under the oxygen-assisted CVD process. Note that much larger sizes of catalyst nanoparticles ( $>2 \mathrm{~nm}$ in diameter) after thermal (VD reaction can be deduced as the original catalyst nanoparticles failed to grow SWNTs but adducted with either amorphous carbons or multiple graphitic layers.

The removal of unreacted catalyst nanoparticles during the oxygen-assisted CVD seems to occur due to the thermal 


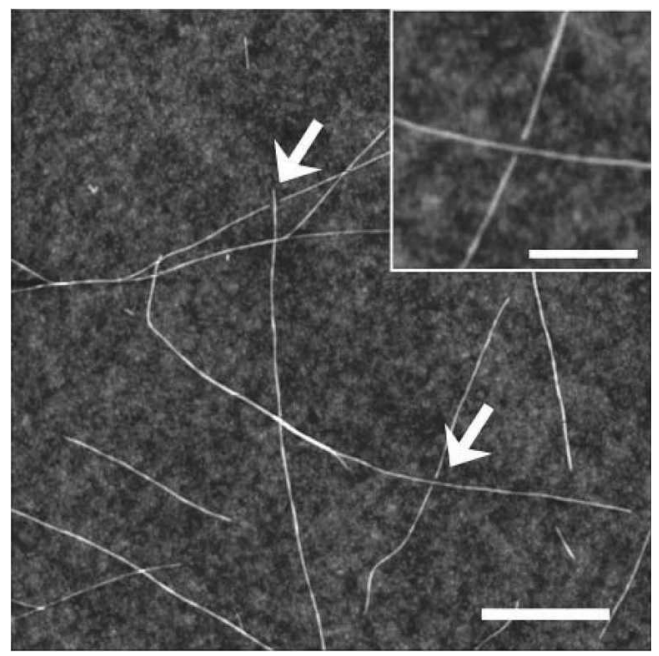

Figure 3. $\Lambda$ representative $\Lambda F M$ image of cut SWNTs (see the arrows) found when nanotubes were grown by oxygen-assisted CVD process (scale bar $=500 \mathrm{~nm}$ ). The inset shows a high magnification inage of the bottom arrow region (scale bar $=200 \mathrm{nun}$ ).

reaction between oxygen and Co nanoparticles. We recently reported that similar thermal reaction between oxygen and Fe nanoparticles adsorbed on $\mathrm{Si}_{\mathrm{SiO}}$ readily removed $\mathrm{Fe}$ nanoparticles from the substrate. In this case, further carbothemnal reduction readily occurred $\left(\mathrm{C}(\mathrm{s})+\mathrm{SiO}_{2}(\mathrm{~s}) \leftrightarrow\right.$ $\mathrm{CO}(\mathrm{g})+\mathrm{SiO}(\mathrm{g}))$ resulting in etching of $\mathrm{SiO}$, layers, ie. formation of nanotrenches. ${ }^{6}$ Although Co does not mediate carbothermal reduction of $\mathrm{SiO}$, layers in the presence of oxygen, ${ }^{6}$ it seems to react with the introduced reacting gas mixtures including oxygen to be removed from the substrate. This phenomenon agrees well with the previously reported effect of oxygen vapor from water ${ }^{+(a)}$ or alcohol, ${ }^{10}$ by which metal catalysts as well as amorphous carbons are efficiently removed during the growth.

Another interesting feature observed from oxygen-assisted CVD growth is that the population of short nanotube is high, and that several nanotubes crossing other nanotubes were cut (Figure 3). It is well known that the surface of SWNT is readily oxidized under oxygen environment at high temperature, ${ }^{11}$ especially at the naturally formed defect sites (e.g. missed carbon to complete benzene structure) or buckled area. In our case, these defective sites seem to be readily eliminated when small amount of oxygen gas was introduced. Although the synthesis of much longer SWNTs is hindered, such phenomenon is quite encouraging in the respect of eliminating most of defective parts of SWNTs.

An apparent improvement of purity was much clearly observed when high yield SWNTs were grown by both processes. In order to grow high yield of SWNTs, high density of Co nanoparticles were deposited on the $\mathrm{Si} / \mathrm{SiO}_{2}$ substrate by prolonged sonication of Co solution over 3 hours (Figure 4(a)). As we observed from low yield samples, the population of unreacted catalyst was much higher when thermal CVD used (Figure 4(b) and (c)). Furthermore, it was clearly seen that amorphous carbons deposited on the sidewalls of SWNTs were a lot less when oxygen was introduced in the growth system.

A successful growth of highly pure SWNTs from Co catalyst nanoparticles by oxygen-assisted CVD was further corroborated by investigating their spectroscopic and electronic transport properties. Figure 5 shows micro-Raman spectra of high yield SWNTs. We were able to estimate diameters of SWNTs by correlating laser wavelength ( 632.8 $\mathrm{nm}, \mathrm{E}_{\mathrm{laser}}=1.96 \mathrm{eV}$ ) and the information related to radial breathing mode $\left(\omega_{\mathrm{RBM}}, 120 \mathrm{~cm}^{-1}<\omega_{\mathrm{RBM}}<250 \mathrm{~cm}^{-1}\right){ }^{12} \mathrm{By}$ using the equation of $\omega_{\mathrm{RMB}}=248 \mathrm{~cm}^{-1} / \mathrm{d}_{\mathrm{l}}$, the characterized resonant nanotube diameters were found to be 1.26 and 1.24 $\mathrm{nm}$ for the SWNTs grown by oxygen-assisted CVD (Figure $5(\mathrm{a}), 197.5 \mathrm{~cm}^{-1}$ ) and thernal CVD process (Figure $5(\mathrm{c})$, $199.8 \mathrm{~cm}^{-1}$ ), respectively, Note that these calculated diameters of SWNTs were a little bigger than the average diameters measured by AFM, and this is due to the limited population of optically allowed electronic transitions by the $632.8 \mathrm{~nm}$ laser source.

The Raman spectra of Figure 5(b) and 5(d) exhibit tangential mode (1450-1650 $\left.\mathrm{cm}^{-1}\right)$ and disorder mode $\left(1250-1350 \mathrm{~cm}^{-1}\right)$ of which ratio indicates the defect extent of nanotubes. An intensity of tangential mode/disorder mode, called 'G/D ratio', is a good indicator for the quality of carbon nanotubes in tems of preservation of $\mathrm{sp}^{2}$ hybridization, and was estimated to be 15.92 for oxygenassisted CVD grown SWNTs (Figure 5(b)) and 16.54 for themnal CVD grown SWNTs (Figure 5(d)). The similar
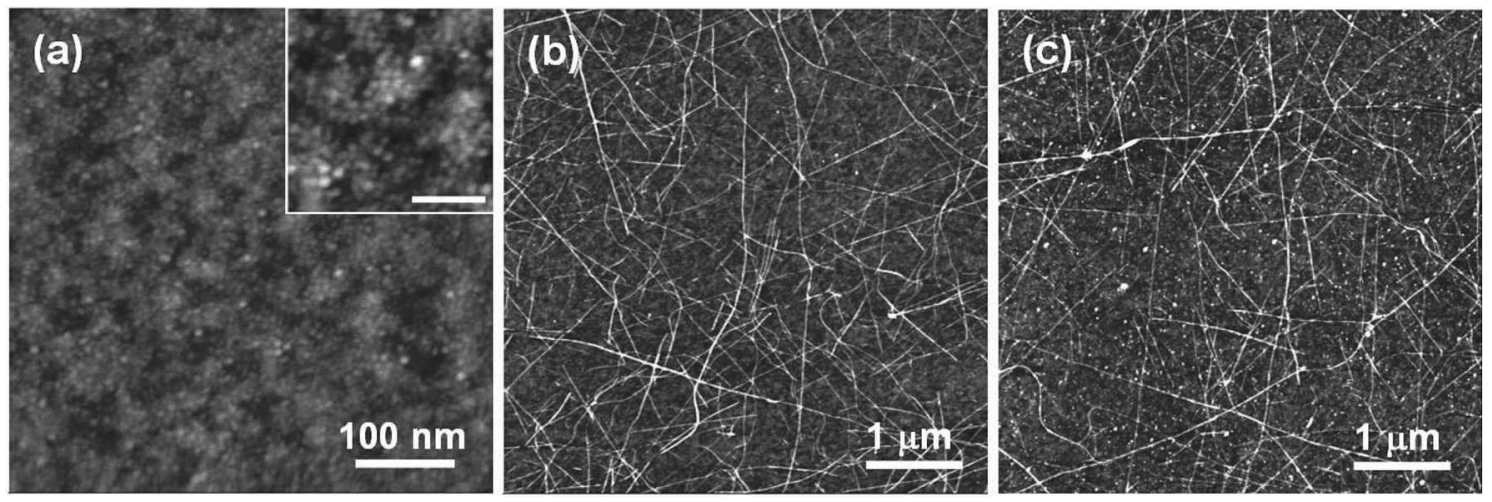

Figure 4. AFM images of (a) high yield of Co nanoparticles, grown SWNTs by (b) oxygen-assisted CVD and (c) thermal CVD processes. Inset of 4(a) is a high magnification image of high population of Co nanoparticles. A scale bar indicates $50 \mathrm{~nm}$. 

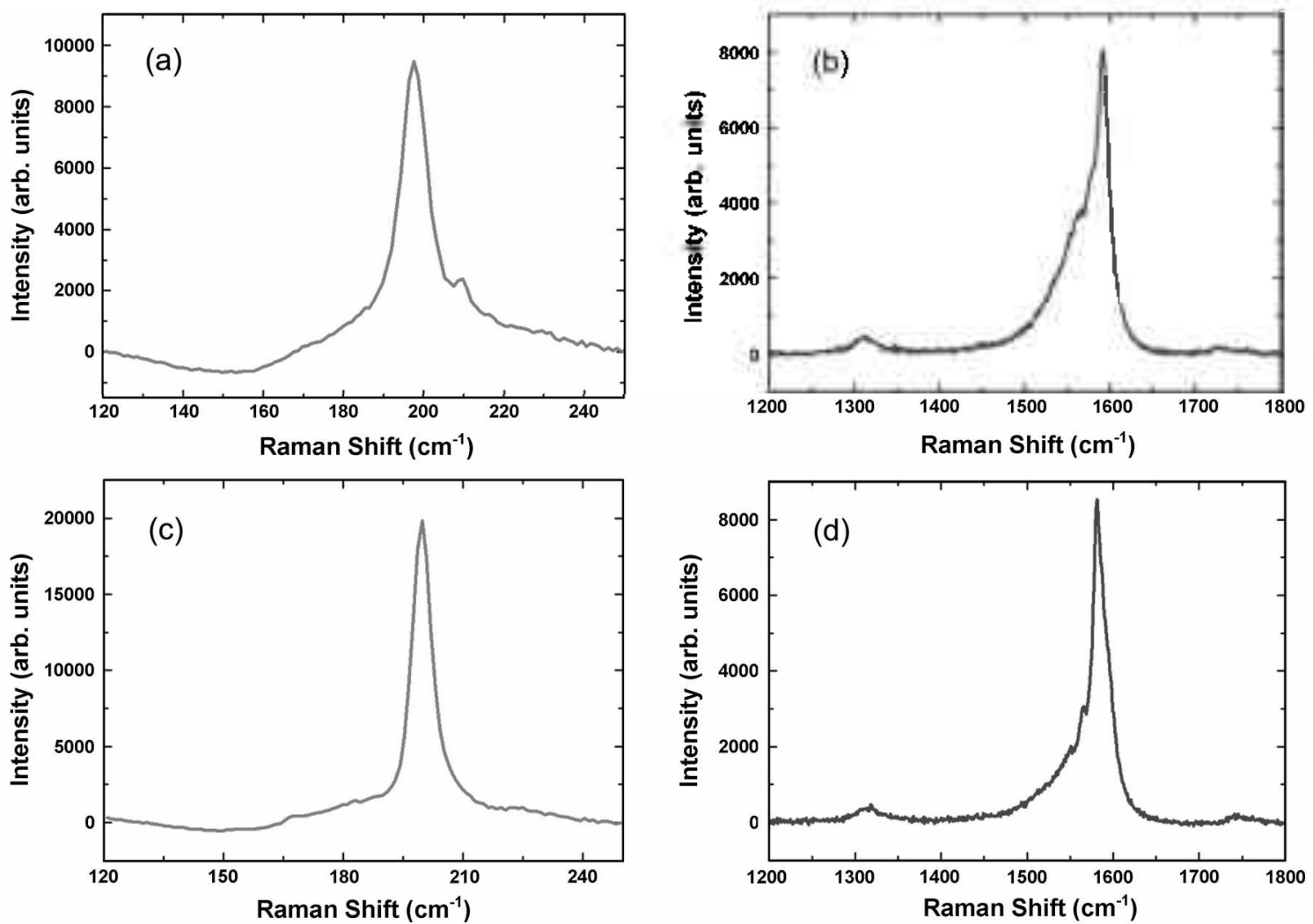

Figure 5. Resonance Ranan spectra of synthesized SWNTs by oxygen-assisted CVD ((a) and (b)) and thernal CVD ((c) and (d)). (a) and (c) show radial breathing modes while (b) and (d) show disorder $\left(1250-1350 \mathrm{~cm}^{-1}\right)$ and tangential mode $\left(1450-1650 \mathrm{~cm}^{-1}\right)$ of high yield of SWNTs. $\left(\mathrm{E}_{\mathrm{l} \text { : } \mathrm{sel}}=1.96 \mathrm{eV}\right)$
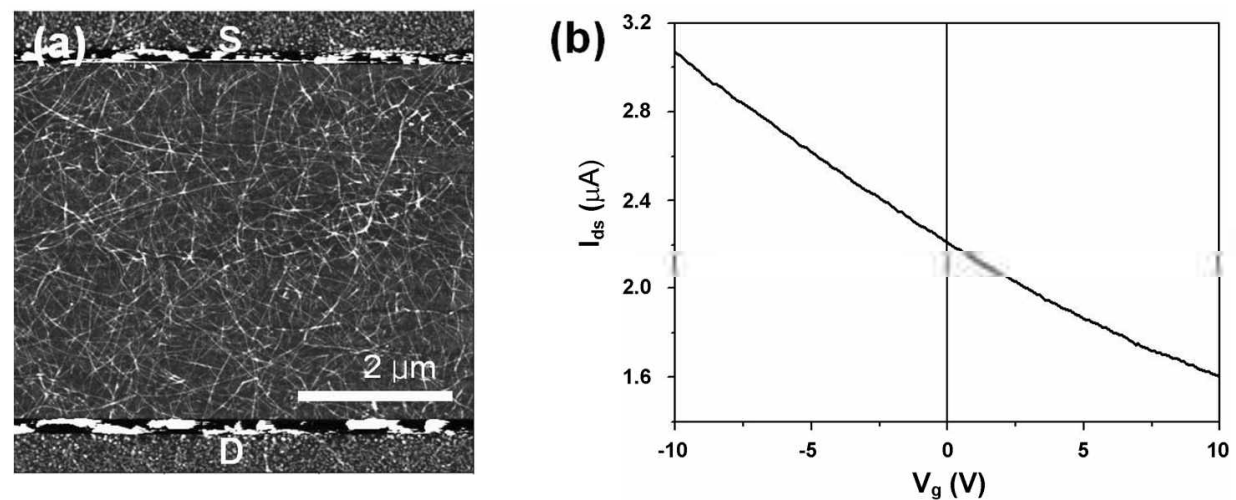

Figure 6. (a) AFM inage of FET device made of SWNTs grown by oxygen-assisted CVD process. The $S$ and $D$ indicate source and drain electrode, respectively. (b) I-V $\mathrm{V}_{L}$ characteristic curve of the SWNT FET device which shows p-type serniconducting transport property (V $\mathrm{V}_{\mathrm{ds}}=$ $10 \mathrm{mV}$ ).

values of $\mathrm{G} / \mathrm{D}$ ratio mean that most parts of the SWNTs grown by oxygen-assisted CVD process are chemically inert, which further stands for that the growth conditions of oxygen-assisted CVD, especially the amounts of oxygen introduced $(-0.1 \%)$ are quite suitable for accomplishing high purity of SWNTs while removing unreacted catalyst nanoparticles with amorphous carbons.

Finally, we characterized the electrical properties of SWNTs grown from Co catalyst nanoparticles by using oxygen-assisted CVD method. We fabricated SWNTs FETs having $5 \mathrm{tan}$ of channel gap with $\mathrm{Au} / \mathrm{Cr}$ layers $(25 / 10 \mathrm{~mm}$ of thickness) as the source and drain electrodes through photolithographic techniques. As shown in Figure 6(a), multiple SWNTs were connected between the source and drain electrodes. We confirmed that the grown SWNTs showed traditional p-type transistor characteristics ${ }^{1(e)}$ by observing over $50 \%$ of current $\left(I_{d s}\right)$ drop upon the gate voltage $\left(\mathrm{V}_{\mathrm{g}}\right)$ sweep from -10 to $+10 \mathrm{~V}$ at $10 \mathrm{mV}$ of bias voltage (Figure $6(b)$ ).

\section{Conclusion}

We successfully synthesized Co nanoparticles with less than $1 \mathrm{~nm}$ of diameters on the $\mathrm{Si} / \mathrm{SiO}_{2}$ substrate. Those 
nanoparticles could grow highly pure SWNTs by introducing $0.1 \%$ amounts of oxygen gas in the conventional thermal CVD system. The small quantity of oxygen flow at high temperature removed amorphous carbons and eliminated unreacted catalysts from the substrate. Raman spectra revealed that most of SWNT surfaces remained intact without severe defects as similar $\mathrm{G} / \mathrm{D}$ ratio was obtained from oxygen-assisted CVD grown SWNTs as the one from thermal CVD grown SWNTs. In addition, it was confirmed that high purity of SWNT-FETs showed a typical p-type transport characteristics.

Acknowledgement. This work was supported by the Nano/Bio Science \& Technology Program of MOST (200600955), SRC/ERC Program (R11-2000-070-070020), Korean Research Foundation (MOEHRD, KRF-2005-005-J13103), and KOSEF (2007-8-1158). Prof. Young Hee Lee and Mr. Sung Jin Kim are thanked for Raman measurements.

\section{References}

1. (a) Kong. J.; Franklin, N.; Chou, C.; Pan, S.; Cho, K. J.; Dai, H. Science 2000, 287, 622. (b) Chen, R. J.; Bangsaruntip, S.; Drouvalakis, K. A.; Kam, N. W. S.; Shim, M.; Li, Y.; Kim, W.; Utz, P. J.; Dai, H. Proc. Natl. Acad. Sci. U.S.A. 2003, 100, 4984. (c) Dillon, A. C.; Jones, K. M.; Bekkedahl, T. A.; Kiang, C. H.; Bethune, D. S.; Heben, M. J. Nature 1997, 386,377 . (d) Fan, S.; Chapline, M. G.; Franklin, N. R.; Tombler, T. W.; Cassell, A. M.; Dai, H. Science 1999, 283, 152. (e) Tans, S.; Verschueren, A.; Dekker, C. Nature 1998, 393, 49.

2. Li, Y.; Kim, W.; Zhang, Y.; Rolandi, M.; Wang. D.; Dai, H. $J$.
Phys. Chem, B 2001, 105, 11424

3. (a) Chiang, I. W.; Brinson, B. E.; Huang, A. Y.; Willis, P. A.; Bronikowski, M. J.; Margrave, J. L.; Smalley, R. E.; Hauge, R. H. J. Phys. Chem. B 2001, 105, 8297. (b) Zimmerman, J. L.; Bradley, R. K.; Huffman, C. B.; Hauge, R. H.; Margrave, J. L. Chem. Mater, 2000, 12, 1361 .

4. (a) Hata, K.; Futaba, D. N.; Mizuno, K.; Namai, T.; Yumura, M.; Jijima, S. Science 2004, 306, 1362. (b) Zhang, G; Mann, D.; Zhang. L.; Javey, A.; Li, Y.; Yenilmez, E.; Wang, Q.; MeVittie, J. P.; Nishi, Y.; Gibbons, J.; Dai, H. Proc. Natl. Acad. Sci. U.S.A. $2005,102,16141$.

5. The 'moderately high yield' means that the population of SWNTs is high enough to form network-type FET devices, but not so high that SWNTs are vertically aligned.

6. Byon, H. R.; Choi, H. C. Nature Nanotech. 2007, 2, 162.

7. Murakami, Y.; Miyauchi, Y.; Chiashi, S.; Maruyama, S. Chen. Phys, Lett. 2003, 377, 49 .

8. (a) Lee, Y.; Song, H. J.; Shin, H. S.; Shin, H. J.; Choi, H. C. Small 2005, 1, 975. (b) Qi, P.; Vermesh, 0.; Grecu, M.; Javey, A.; Wang, Q.; Dai, H. Nano Lett. 2003, 3, 347.

9. (a) Choi, H. C.; Kim, W.; Wang, D.; Dai, H. J. Phys. Chem. B 2002, 106, 12361. (b) Choi, H. C.; Kundaria, S.; Wang, D.; Javey, A.; Wang, Q.; Rolandi, M.; Dai, H, Nano Lett. 2003, 3, 157. (c) Yang, H. J.; Song, H. J.; Shin, H. J.; Choi, H. C. Langmuir 2005, 21,9098 .

10. (a) Murakami, Y.; Chiashi, S.; Miyauchi, Y.; Hu, M.; Ogura, M.; Koubo, T.; Maruyama, S. Chen. Phys. Left. 2004, 385, 298. (b) Sato, H.; Hata, K.; Hiasa, K.; Saito, Y. J. Vac. Sci. Technol. B $2007,25,579$.

I1. Li, J.-L.; Kudin, K. N.; Mcallișter, M. J.; Prud'homme, R. K.; Aksay, I. A.; Car, R. Phys. Rev, Lett. 2006, 96, 176101.

12. Jorio, A.; Pimenta, M. A.; Souza Filho, A. G.; Saito, R.; Dresselhaus, G.; Dresselhaus, M. S. New J. Phys. 2003, 5, 139.

13. Kataura, H.; Kumazawa, Y.; Maniwa, Y.; Uemezu, I.; Suzuki, S.; Ohisuka, Y.; Achiba, Y. Synth. Met. 1999, 103, 2555. 